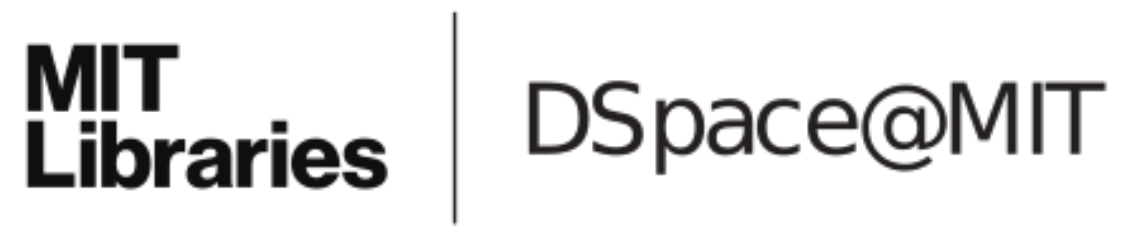

MIT Open Access Articles

Formulations for trans-tympanic antibiotic delivery

The MIT Faculty has made this article openly available. Please share how this access benefits you. Your story matters.

Citation: Khoo, Xiaojuan et al., "Formulations for trans-tympanic antibiotic delivery." Biomaterials 34, 4 (January 2013): p. 1281-88 doi. 10.1016/j.biomaterials.2012.10.025 (c2012 Authors

As Published: https://dx.doi.org/10.1016/j.biomaterials.2012.10.025

Publisher: Elsevier BV

Persistent URL: https://hdl.handle.net/1721.1/126215

Version: Author's final manuscript: final author's manuscript post peer review, without publisher's formatting or copy editing

Terms of use: Creative Commons Attribution-NonCommercial-NoDerivs License 


\title{
Formulations for Trans-Tympanic Antibiotic Delivery
}

\author{
Xiaojuan Khoo ${ }^{3,4, \dagger}$, Emmanuel J. Simons ${ }^{1,4, \dagger}$, Homer H. Chiang ${ }^{4}$, Julia M. Hickey ${ }^{4}$, \\ Vishakha Sabharwal ${ }^{5}$, Stephen I. Pelton ${ }^{5}$, John J. Rosowski ${ }^{1,2}$, Robert Langer ${ }^{1,3}$, and \\ Daniel S. Kohane ${ }^{1,4,{ }^{*}}$ \\ ${ }^{1}$ Harvard-MIT Division of Health Sciences and Technology, Cambridge, MA 02139, USA \\ ${ }^{2}$ Department of Otology and Laryngology, Eaton Peabody Laboratory, Massachusetts Eye and \\ Ear Infirmary, Boston, MA 02114, USA
}

${ }^{3}$ Department of Chemical Engineering, Massachusetts Institute of Technology, Cambridge, MA 02139, USA

${ }^{4}$ Laboratory for Biomaterials and Drug Delivery, Department of Anesthesiology, Division of Critical Care Medicine, Children's Hospital Boston, Harvard Medical School, 300 Longwood Ave., Boston, MA 02115, USA

${ }^{5}$ Division of Pediatric Infectious Diseases, Maxwell Finland Laboratory for Infectious Diseases, Boston Medical Center, Boston, MA 02118, USA

\section{Introduction}

Acute otitis media (AOM) is the most frequently diagnosed illness among children in the United States, accounting for over 20 million physician visits each year [1,2]. Recurrence of disease is also common, with one third of all children in the U.S. having 6 or more episodes of AOM by age 7 [3]. The current treatment regimen for AOM typically consists of a tenday course of broad spectrum oral antibiotics [4]. Systemic administration of broadspectrum antibiotics against a disease with such high prevalence and recurrence is believed to be partially responsible for the emergence of drug-resistant strains of pathogenic bacteria [5].

Ototopical drug delivery presents a highly promising alternative to oral antibiotics for the administration of therapeutics to the middle ear. The localized delivery of antibiotics across the tympanic membrane (TM) directly to the middle ear could potentially enhance the local bioavailability of drug while minimizing antibiotic exposure of normal flora elsewhere in the body, which together reduce the selective pressures responsible for antibiotic resistance. The utility of ototopical administration is seen in the effectiveness of antibiotic eardrops in patients with punctured TMs or tympanostomy tubes [6]. However, the significant penetration barrier provided by the stratum corneum (SC) has restricted the clinical use of such drops to patients with non-intact TMs. Strategies to overcome the SC barrier, including chemical additives, iontophoresis and micro-injection, have been extensively explored for use in transdermal drug delivery applications [7]. Chemical permeation enhancers (CPEs), in

\footnotetext{
(C) 2012 Elsevier Ltd. All rights reserved.

*Corresponding author: Daniel.Kohane@childrens.harvard.edu.

$\dagger$ Equal contribution
}

Publisher's Disclaimer: This is a PDF file of an unedited manuscript that has been accepted for publication. As a service to our customers we are providing this early version of the manuscript. The manuscript will undergo copyediting, typesetting, and review of the resulting proof before it is published in its final citable form. Please note that during the production process errors may be discovered which could affect the content, and all legal disclaimers that apply to the journal pertain. 
particular, have emerged as an effective means of enhancing small molecule flux across the skin [8-11]. By reversibly increasing the permeability of the stratum corneum, CPEs greatly improve the trans-dermal delivery of molecules that would otherwise permeate poorly, for a wide range of cosmetic and dermatological applications [7, 12-14]. Given the structural similarities between skin and the TM, a formulation combining both CPEs and antibiotics could potentially enhance drug flux across the intact TM into the middle ear.

A clinically effective local delivery system that reproduces the effect of an extended systemic drug regimen should not only increase drug flux across the TM, but also maintain it over a prolonged period. Additionally, the drug regimen would ideally be delivered in a single dose, as compliance with multi-dose regimens can be difficult, particularly in the treatment of uncooperative young patients. These design criteria are particularly important in the case of treatment of AOM, where otoscopy would be required for placement; it could be done at the time of diagnosis, but would be impractical thereafter. A delivery vehicle capable of holding both antibiotic and CPE in place at the TM would be ideal for this purpose. In situ gelling systems, with their ease of application and ability to conform to a delivery site, would be most suitable for ototopical use. Poloxamers (tri-block copolymers of polypropylene and polyethylene) have been used for the controlled release of therapeutic compounds due to their thermoreversible gelation behavior and established safety in FDAapproved preparations [15-17]. Solutions of poloxamer 407 (P407) undergo a liquid-gel transition below body temperature, which could allow them to be administered in the form of a liquid ear drop into the ear canal, but gel rapidly in situ upon contact with the TM. The purpose of incorporating antibiotics and CPEs into such a gel system is that it will increase therapeutic efficacy by maximizing TM contact time and prolonging drug release (Figure 1A).

The objective of this study was to develop and evaluate formulations for the sustained transtympanic delivery of antibiotics. Ciprofloxacin, a synthetic fluoroquinolone antibiotic, was selected for these experiments because of its known activity against Haemophilus influenzae (H. flu) and Streptococcus pneumoniae (S. pneumo), its low molecular weight and moderate lipophilicity, and its established ototopical use in children with AOM with tympanostomy tubes $[18,19]$. To enhance drug flux across the intact TM, we incorporated CPEs (sodium dodecyl sulfate, limonene, and bupivacaine) into formulations and examined the resultant changes in trans-tympanic drug permeability across native chinchilla TMs. We further investigated the effect of the gel-enhancer formulations on hearing thresholds via in vivo auditory brainstem response (ABR) measurements in chinchillas, and examined their in vivo tissue toxicity.

\section{Materials and Methods}

\subsection{Materials}

Ciprofloxacin hydrochloride (1\% w/v; MW $=331.4 \mathrm{Da}, \log \mathrm{P}=0.28[19])$ was purchased from Bayer HealthCare (West Haven, CT). Poloxamer 407 (P407 or Pluronic F-127®) and all other chemicals and reagents were obtained from Sigma-Aldrich (St. Louis, MO), unless otherwise specified.

\subsection{Animal maintenance}

Healthy adult male chinchillas weighing 550 to $650 \mathrm{~g}$ were purchased from Ryerson Chinchilla Ranch (Plymouth, $\mathrm{OH}$ ) and cared for in accordance with protocols approved institutionally and nationally. Experiments were carried out in accordance with the Massachusetts Eye and Ear Infirmary (MEEI) Animal Use Guidelines and approved by the 
MEEI and Massachusetts Institute of Technology (MIT) Institutional Animal Care and Use Committees.

\subsection{Hydrogel formulation}

Hydrogels were prepared using the "cold method" [20]. Briefly, a weighed amount of P407 was added to $\mathrm{dH}_{2} \mathrm{O}$ or an aqueous solution of $1 \%$ cipro in a $20 \mathrm{~mL}$ glass scintillation vial and mixed overnight at $4^{\circ} \mathrm{C}$ on a magnetic stir plate to effect complete solubilization. The P407 concentration in gels is expressed as the weight percentage (wt\%), unless specified otherwise. To prepare CPE-loaded hydrogels, appropriate volumes of CPEs were slowly added to the poloxamer-drug mixture under gentle stirring. Resultant formulations were liquid at $4^{\circ} \mathrm{C}$. CPEs (sodium dodecyl sulfate (SDS); limonene (LIM); bupivacaine (BPV)) representing different chemical classes were used in this study, and, unless otherwise stated, were fixed at $1 \%$ SDS, $2 \%$ LIM and $0.5 \%$ BPV. These concentrations were selected based on those reported to be effective and minimally toxic in previous transdermal studies [11] or clinical practice. A combination of all three CPEs is represented as 3CPE.

\subsection{Study of gelation properties}

The gelation temperature $\left(\mathrm{T}_{\mathrm{gel}}\right)$ of each $\mathrm{P} 407$ hydrogel formulation was measured using a modification of a published method [21]. $200 \mu \mathrm{L}$ of each formulation was added to a glass HPLC sample vial with a magnetic stir bar and immersed in a low-temperature water bath on a heated stir plate. Each vial was gradually heated from $20^{\circ} \mathrm{C}$ to $40^{\circ} \mathrm{C}$ at the rate of $1{ }^{\circ} \mathrm{C} /$ min with continuous stirring at $200 \mathrm{rpm}$ and the temperature at which the stir bar stopped rotating was recorded as the $\mathrm{T}_{\text {gel }}$. The time to gelation was also noted. Measurements were performed in quadruplicate.

\subsection{In vitro release studies}

The release of ciprofloxacin from $\mathrm{P} 407$ gels was measured using a diffusion system. Transwell ${ }^{\circ}$ membrane inserts $\left(0.4 \mu \mathrm{m}\right.$ pore size, $1.1 \mathrm{~cm}^{2}$ area; Costar, Cambridge, MA) and 24-well culture plates were employed as the donor and acceptor chambers, respectively. 200 $\mu \mathrm{L}$ of each $18 \% \mathrm{P} 407$ formulation loaded with $2 \mathrm{mg}$ ciprofloxacin was pipetted directly onto pre-warmed filter inserts to obtain a solid hydrogel. Filter inserts (donor compartments) with formed gels were suspended in wells (acceptor compartments) filled with pre-warmed phosphate buffered saline (PBS) and the plates then incubated in a $37^{\circ} \mathrm{C}$ oven. At each time point $(0.5,1,2,6,24$, and $48 \mathrm{hr}$.), $1 \mathrm{~mL}$ aliquots of the PBS receiving media were sampled and inserts sequentially moved into a new well with fresh PBS. Aliquots were resuspended in 70:30 acetonitrile/PBS to ensure total drug dissolution. Sample aliquots were chromatographically analyzed with HPLC to determine ciprofloxacin concentrations $(\lambda=$ $275 \mathrm{~nm}$ ). Experiments were performed in quadruplicate.

\subsection{Ciprofloxacin measurement}

Ciprofloxacin levels were determined using an Agilent 1100 high-performance liquid chromatography (HPLC) system.10- $\mu \mathrm{L}$ samples were injected onto an Agilent Poroshell 120 EC-C18 column $(4.6 \mathrm{~mm} \times 150 \mathrm{~mm}, 2.7 \mu \mathrm{m}$ particle size. The mobile phase used was 80:20 acetonitrile: $0.1 \%$ trifluoroacetic acid $\mathrm{pH}=2.0$ ) at a flow rate of $1 \mathrm{~mL} / \mathrm{min}$.

Ciprofloxacin was detected by UV absorbance at $275 \mathrm{~nm}$ wavelength. Separate dilution standards were prepared by diluting $1 \%$ cipro in 70:30 acetonitrile:PBS, $0.01 \%$ to $1.0 \times$ $10^{-4} \%(\mathrm{w} / \mathrm{v})$, on the day of analysis.

\subsection{Tympanic membrane harvesting}

For ex vivo drug permeation studies, TMs were extracted from euthanized chinchillas. Chinchillas were placed under deep general anesthesia by the intramuscular administration 
of ketamine $(30 \mathrm{mg} / \mathrm{kg})$ and xylazine $(4 \mathrm{mg} / \mathrm{kg})$, and then euthanized with intracardiac administration of pentobarbital $(100 \mathrm{mg} / \mathrm{kg})$. Euthanized animals were decapitated and the auditory bullae removed from the rest of the skull. Bullae were excised and the tympanic membrane (TM) and surrounding bony annulus carefully extracted. The TMs (with the imbedded manubrium of the malleus) were maintained intact within the tympanic ring and exposed on both lateral and medial surfaces. Immediately following dissection, TMs were inspected visually with a stereo dissection microscope under 10X magnification for perforations.

\subsection{Tympanic membrane (TM) electrical resistance}

The electrical impedance of excised chinchilla TMs was measured as previously described for skin [22], with the following modifications. Excised chinchilla TMs and attached tympanic rings were placed upright in a 12-well plate, with the TM surface perpendicular to the well base and the EAM longitudinal axis parallel to the well walls. Ag-Cl electrodes (In Vivo Metrics, Healdsburg, CA) were placed on either side of the chinchilla TM, in the donor and receiving media, and a signal generator (Hewlett Packard, HP 33120A) provided a 100 $\mathrm{mV}$ (rms) AC voltage for 5 seconds at $10 \mathrm{~Hz}, 100 \mathrm{~Hz}, 1 \mathrm{kHz}$, and $10 \mathrm{kHz}$. The current passing through the membrane was measured with a Fluke Multimeter (Model 139, Fluke Corporation), and the electrical impedance was approximated using Ohm's Law from the current obtained with the $10 \mathrm{~Hz}$ signal. Background resistance measurements of PBS alone were made separately and subtracted from the initial resistance calculation to yield the membrane resistance. Any sample with an initial resistivity (electrical resistance * exposed area) of $<18 \mathrm{kOhm} * \mathrm{~cm}^{2}$ was considered damaged and replaced with an intact sample [23, 24]. Following the final time point in the extracted TM experiments, the TM surface was covered with a thin rubber disc and silicone adhesive, and the electrical resistance of the surrounding tympanic ring were measured and similarly subtracted from the initial TM + EAM resistance calculation.

\subsection{Ex vivo ciprofloxacin permeation studies}

Trans-tympanic flux experiments were performed to examine the effect of single and combination CPEs (no gel) on ciprofloxacin diffusion across the chinchilla TM. Excised chinchilla bullae were placed upright in a 12-well plate, with the TM surface perpendicular to the well base and the EAM longitudinal axis parallel to the well walls. A $3 \mathrm{~mL}$ volume of PBS was added to the well, so that the entire medial surface of the TM was submerged and $200 \mu \mathrm{L}$ of the test CPE solution pipetted into the EAM to cover the lateral TM surface. Plates were stored at $37^{\circ} \mathrm{C}$. At pre-determined time points $(0.5,1,2,6,12,24,48$, and 120 hours post treatment administration), a $1 \mathrm{~mL}$ sample from the $1 \mathrm{~mL}$ receiving chamber was removed, diluted to 70:30 acetonitrile:buffer, filtered, and transferred to an HPLC vial. Bullae were sequentially moved to fresh wells containing $3 \mathrm{~mL}$ PBS. The samples were then chromatographically analyzed with HPLC to determine the ciprofloxacin concentrations.

Ciprofloxacin flux from hydrogels was similarly evaluated in order to determine the effects, if any, of incorporation into a P407 gel system. $200 \mu \mathrm{L}$ of liquid 18\% P407 or 18\% P407$\mathrm{CPE}$ formulations were pipetted into the EAM (warmed to $37^{\circ} \mathrm{C}$ ) to form a gel plug. Aliquots were removed and evaluated as above. Experiments were performed in quadruplicate and only harvested TMs with $R A \geq 18 \mathrm{kOhm} * \mathrm{~cm}^{2}$ were used.

\subsection{Auditory brainstem response (ABR) measurements}

$\mathrm{ABR}$ experiments were conducted with a custom-designed stimulus generation and measurement system built around National Instruments (Austin, TX) software (Lab View) and hardware. The hardware included a GPIB controller and an ADC board. A custom LabView program computed the stimuli, and downloaded the stimuli to a programmable 
stimulus generator (Hewlett Packard 33120A). The stimulus was then filtered by an antialiasing filter (KrohnHite 3901) and attenuated (Tucker-Davis Technologies). The filter and the attenuator were controlled by the LabView software. Simultaneous with stimulus output, the 2 ADC channels sampled the amplified ABR signal and the output of a microphone sealed in the ear canal of the animal.

The attenuated stimulus was played through a hearing-aid earphone placed within the intact ear canal of adult female chinchillas (400-600 g) anesthetized by IP administration of sodium pentobarbital $(60 \mathrm{mg} / \mathrm{kg})$. The earphone coupler included a microphone that monitored the sound stimulus level. ABRs, obtained in a sound-attenuating booth, were measured with a differential amplifier with a gain of 10,000 and a measurement bandwidth of $100 \mathrm{~Hz}$ to $3 \mathrm{kHz}$. The measurements were obtained from the positive electrode in the muscle behind the measured ear; the negative electrode was at the cranial vertex, and the ground electrode behind the contralateral ear. After obtaining baseline measurements, $200 \mu \mathrm{L}$ of $18 \% \mathrm{P} 407$ gel was applied to the ear canal and the same measurements immediately performed to examine the resultant changes, if any, in auditory sensitivity thresholds.

The acoustic stimuli were pairs of 20-ms tone bursts of opposite polarity. The frequency of the bursts increased from $500 \mathrm{~Hz}$ to $16 \mathrm{kHz}$ in octave steps. Each burst was sine windowed, with $40 \mathrm{~ms}$ between the two bursts. ABR responses to 250 pairs of stimuli were averaged at each stimulus level. The ABR response was computed from the sum of the averaged response to the two different polarities. Stimulus level was varied in $10 \mathrm{~dB}$ steps. A visual judgment of threshold at each stimulus frequency was determined post-measurement in a blinded fashion. After ABR measurements, the animals were monitored till they recovered from anesthesia and were returned to the colony.

\subsection{Histopathology}

P407 formulations containing ciprofloxacin and CPEs were administered to the ear canals of live chinchillas. Seven days later, they were euthanized as described above. Following sacrifice, the bullae were excised as described above and the bone around the TM carefully removed leaving an intact TM, exposed on both lateral and medial surfaces, within the tympanic ring. Excised TMs were immediately fixed in $10 \%$ formalin overnight, then decalcified, embedded in paraffin, sectioned ( $5 \mu \mathrm{m}$ thick) and stained with hematoxylin and eosin by the Department of Comparative Medicine (DCM) at MIT (fee for service), using standard techniques. All stained specimens were evaluated under light microscopy (Olympus FSX-100) by a blinded histologist.

\subsection{Statistical analysis}

All data populations were first assessed to determine which were normally distributed. Normally distributed data are presented as means with standard deviation, and compared by t-tests and analysis of variance (ANOVA), where specified; data not normally distributed are presented as medians with 25th and 75th percentiles and compared by Mann-Whitney Utest. Statistical significance, for both parametric and non-parametric tests, was defined as $p<$ 0.05 .

\section{Results}

\subsection{Gelation studies}

The thermal gelation behavior of ototopical formulations was investigated to establish the P407 concentration that would provide a rapid and reproducible liquid-gel transition between room and body temperatures while loaded with ciprofloxacin and CPEs (Table 1). 
Each formulation was a viscous liquid at storage temperature $\left(4^{\circ} \mathrm{C}\right)$, formed a semisolid gel above its liquid-gel transition temperature $\left(\mathrm{T}_{\text {gel }}\right)$, and returned to the liquid state below $\mathrm{T}_{\text {gel. }}$. $\mathrm{T}_{\text {gel }}$ decreased with increasing P407 concentration; $18 \%$ and 20\% formulations formed soft, clear gels at $37^{\circ} \mathrm{C}$ that did not run when inverted (Figure 1B). The incorporation of all three CPEs (3CPE) into formulations produced a large decrease in $\mathrm{T}_{\text {gel }}$ at all three $\mathrm{P} 407$ concentrations tested $(\mathrm{p}<0.001$, compared to gels without $3 \mathrm{CPE})$; the $\mathrm{T}_{\text {gel }}$ of an $18 \% \mathrm{P} 407$ formulation decreased from $38.5^{\circ} \mathrm{C}$ to $24.5^{\circ} \mathrm{C}$ upon the incorporation of 3CPE. Gelation times of gels at $37^{\circ} \mathrm{C}$ ranged from 9 to 42 seconds, with the exception of $16 \% \mathrm{P} 407$ formulations, which took 90 seconds or longer (Table 1). We selected the P407 concentration of $18 \%$ for subsequent ciprofloxacin release and permeability studies.

\subsection{Effect of CPEs on ciprofloxacin permeation across the TM}

We evaluated the effect of CPEs on ciprofloxacin permeation across the chinchilla TM at 37 ${ }^{\circ} \mathrm{C}$ (Figure 2). The initial amount of ciprofloxacin applied to the donor surface of the TM was kept constant at $2 \mathrm{mg}$ for all formulations (i.e., $0.2 \mathrm{~mL}$ of a $1 \%$ solution). In the absence of CPEs, ciprofloxacin permeation was undetectable up to 12 hours. At 24 hours, $109 \mu \mathrm{g}$, or $5.5 \%$ of the starting drug load, had permeated the TM; at 48 hours $364 \mu \mathrm{g}(18 \%)$ had done so. The addition of limonene (LIM) accelerated drug permeation; ciprofloxacin was detected in the receiving buffer in as little as 1-2 hours. A two-to-three-fold concentration-dependent increase in ciprofloxacin transfer $(\mathrm{p}=0.014,0.029$ and 0.029 for $1 \%, 2 \%$ and $4 \%$ limonene, respectively, compared to ciprofloxacin alone) at 48 hours was also achieved.

An alternative to increasing the concentration of a single CPE is to employ combinations of two or more CPEs at lower concentrations. This approach has been demonstrated to achieve efficacy with reduced toxicities [25-31]. Here, we used three different CPEs (0.5\% bupivacaine w/v (BPV), $1 \%$ sodium dodecyl sulfate w/v (SDS), and 2\% limonene (LIM) v/ v) with varying modes of action in enhancing flux [32]. The median ciprofloxacin transfer across the TM in the absence of CPEs was again low, with $152 \mu \mathrm{g}$ (or $7.6 \%$ of the original drug load) transferred at 24 hours, and approximately $445 \mu \mathrm{g}$ (or $22 \%$ ) by the end of 48 hours (Figure 3). The ciprofloxacin permeation produced by the three CPEs in combination was larger than that produced by individual CPEs. A combination of all three CPEs (“3CPE”) was used for all subsequent studies.

\subsection{In vitro drug release from P407}

Ciprofloxacin release from P407 gels with and without CPEs into aqueous receiving buffer was measured at $37{ }^{\circ} \mathrm{C}$ (Figure 4). The in vitro release profile revealed a rapid release of ciprofloxacin from gels. Similar cumulative release profiles were observed with and without CPEs, although the rate of ciprofloxacin release was slightly slower in the presence of 3CPE at the earlier time points (e.g., at 2 and 6 hours, $p=0.029$ ), suggesting the presence of CPEdrug and/or CPE-gel interactions. Drug release reached a plateau by approximately $24 \mathrm{~h}$.

\subsection{Ex vivo TM permeation from hydrogels}

Drug-loaded 18\% P407 formulations formed soft, clear gels when deposited on harvested chinchilla TMs at $37^{\circ} \mathrm{C}$. The presence of the hydrogel matrix slowed the trans-tympanic transfer of ciprofloxacin (compared to that from ciprofloxacin solution), with ciprofloxacin levels remaining at undetectable levels for at least 12 hours. The addition of $3 \mathrm{CPE}$ increased flux, so that $3 \mu \mathrm{g}$ of ciprofloxacin crossed the TM after 6 hours and $14 \mu \mathrm{g}$ crossed after 12 hours (Figure 5, inset) ( $p=0.0062$ compared with gel without $3 \mathrm{CPE}$ ). This increase was seen at all time points, with $3 \mathrm{CPE}$ almost doubling the amount of ciprofloxacin crossing the TM in 120 hours ( $812 \mu \mathrm{g}$ vs. $441 \mu \mathrm{g}$; $\mathrm{p}=0.018$ ). Permeation across the TM from hydrogel-3CPE formulations was much slower than from the $3 \mathrm{CPE}$ liquid (no hydrogel) 
solution (Figure 4). Ciprofloxacin permeation across the TM from gels was observed for at least 120 hours.

\subsection{ABR measurements}

Acoustic brain response (ABR) measurements in live chinchillas were used to noninvasively assess impairment of hearing sensitivity from application of the gel-enhancer formulation to the TM. Baseline ABRs before application of gels showed the normal pattern of increasing sensitivity (decreased thresholds) as frequency increased from $500-\mathrm{Hz}$ to $4-$ $\mathrm{kHz}$ tones, and a subsequent increase in thresholds from 4 to $16 \mathrm{kHz}$ (data not shown). Visual inspection under 5x magnification confirmed gel placement and the formation of a thin, near-uniform layer covering the entire TM surface. The gel-enhancer formulation had no significant effect on hearing upon application, with post-application thresholds comparable to the pre-application baseline measurements in the same animals (Figure 6).

\subsection{Histological analyses}

Tissue response of chinchilla TM to the gel formulations was determined 7 days after administration in vivo. Normal, untreated TMs (Figure 7A) were consistently 10-20 $\mu \mathrm{m}$ thick with three distinct layers: the outer stratified squamous epithelium, an interstitial connective tissue layer, and the inner mucosal epithelium. TMs extracted after 7 days of untreated $H$. influenzae infection were approximately five times thicker (Figure 7B) and exhibited a prominent inflammatory response with diffuse edema and dense infiltration by neutrophils. In comparison, uninfected TMs exposed to ciprofloxacin-loaded gels without 3CPE were mildly edematous and 30-50\% thicker than normal tissue, but did not show any inflammatory cells (Figures 7C and 7D). Slightly more pronounced edema was seen in tissues exposed to ciprofloxacin-loaded gels with 3CPE, but there were no other signs of tissue injury, necrosis, or inflammation were observed. These results demonstrate a generally benign tissue response to the gel-enhancer formulations.

\section{Discussion}

Sustained delivery of antibiotics directly to the middle ear offers several advantages over systemic administration routes. These include reduced side effects, lower frequency of administration, improved patient compliance, and less development of antimicrobial resistance [33-35]. Hydrogel systems have seen extensive use for the delivery of drugs and other small molecules, including anesthetics, antifungals, and chemotherapy,to in vivo targets [33, 34, 36-38]. Hyaluronic acid and fibrin-based hydrogels have been injected directly across the TM to achieve sustained gentamicin delivery in the treatment of inner ear disease $[39,40]$. However, otic gel preparations for non-invasive antibiotic delivery across the TM to the middle ear have not been explored due to the impermeability of the intact TM to all but the smallest, moderately hydrophobic molecules [33, 41-43], and also because of the lack of appropriate model systems in which to easily measure trans-tympanic drug flux. One of the key endeavors of this work was to establish a reproducible ex vivo model using harvested chinchilla TMs to investigate the effects of CPEs on permeant drug flux across the intact TM. This protocol involved a surgical method that avoided TM injury during extraction, and a method to measure electrical resistance across the TM to demonstrate TM integrity. We used this self-contained setup in lieu of a conventional diffusion cell, which could deform or damage the TM. In the present study, we have demonstrated that CPEs can markedly enhance the transfer of the antibiotic ciprofloxacin across the TM, and that this enhanced permeation can be maintained after incorporation into an in situ gelling hydrogel matrix intended to maintain the drug at the TM and reduce the drug dosing frequency. The extent and rate of trans-TM ciprofloxacin permeation from poloxamer gel carriers were 
substantially reduced as compared to those observed from otherwise identical liquid (no hydrogel) mixtures, presumably by slowing diffusion.

One major consideration with the use of CPEs is the balance between efficacy and toxicity, as different mechanisms of stratum corneum disruption result in different toxicity-efficacy profiles amongst CPE classes [9]. Limonene has high enhancement properties and synergistic interaction with other CPEs [11, 48], as do anionic surfactants such as sodium octyl sulfate and amino esters such as tetracaine [11, 48, 49]. Hence, in this study, we selected one CPE from each of these three chemical classes: sodium dodecyl sulfate (SDS; surfactant), limonene (LIM; terpene), and bupivacaine (BPV; amino amide). CPE concentrations were based upon those reported to be effective and minimally toxic in previous transdermal studies $[11,50]$ or clinical practice [51, 52]. Increasing the concentration of a single $\mathrm{CPE}$, in this case limonene, led to a corresponding increase in the total amount of ciprofloxacin transferred across the TM at 48 hours (Figure 2). Similarly, a formulation combining all three CPEs (3CPE) exhibited higher ciprofloxacin permeation after 48 hours compared to formulations with individual CPEs (Figure 3). These results suggest that a combination of multiple CPEs can be used to enhance permeability [28, 53]. Bupivacaine, an amino amide local anesthetic, could also have potential clinical benefit in treating OM-associated otalgia [11, 54].

$18 \% \mathrm{P} 407$ formulations exhibited fast and reproducible thermoreversible gelation. [55, 56] Salts and other additives, by perturbing P407 aggregation, have been shown to dramatically alter both the gelation properties [57-59] and release kinetics [60,61] of poloxamer vehicles. In our multicomponent formulations, gelation was not inhibited by the addition of CPEs, although a decrease in $\mathrm{T}_{\text {gel }}$ was observed. The short gelation times of the gel-3CPE system are favorable from a practical standpoint. The use of a gel carrier also enables prolonged and continuous delivery of active therapeutics to the middle ear following a single administration.

Incorporation of $3 \mathrm{CPE}$ within gels caused a slight decrease in the rate of ciprofloxacin release into an aqueous buffered solution in vitro, suggesting the presence of CPE-gel or CPE-drug interactions. However, the 3CPE increased both the rate and magnitude of ciprofloxacin transfer across the TM ex vivo. This discrepancy between in vitro and ex vivo findings suggests that it is TM permeability, rather than the rate of drug elution from gels, that determines the extent of ciprofloxacin flux across the TM. Similar to transdermal drug delivery systems, CPEs most likely disrupt the stratum corneum barrier of the TM, thus increasing its permeability to ciprofloxacin [49]. The inclusion of CPEs in our formulations is crucial to enhancing the TM permeability to allow for therapeutic levels ciprofloxacin in the middle ear and achieve effective bacterial killing.

Biocompatibility was an important issue to address before initiating further studies to prove efficacy in an animal model of otitis media. Histological analysis indicated a benign tissue reaction after prolonged TM exposure to the gel-3CPE formulation containing ciprofloxacin (Figure 7D). The non-ototoxicity of ciprofloxacin and other fluoroquinolone topical antibiotics is known $[62,63]$. The ABR studies addressed the important issue of whether hydrogel application would impede or distort hearing, which would not be ideal from a clinical point of view. ABR measurements detected small changes in auditory sensitivity that were within the normal range (Figure 6). This suggests that hearing sensitivities remained largely unchanged before and after gel application, possibly due to the thin, uniform, and hydrated nature of the gel coating on the TM.

Our study demonstrates the feasibility of sustained delivery of antibiotics from a hydrogel reservoir to achieve significant drug diffusion across the intact TM into the middle ear. 
Given the similarities between the human and chinchilla ear in terms of TM size, middle ear structure, and auditory range $[64,65]$, the gel-enhancer formulations have potential for use in the ototopical delivery of active antibiotics across intact TMs in human subjects. The low in vivo toxicity associated of these formulations also leaves room for increased CPE concentrations, if necessary.

\section{Conclusion}

We have developed a therapeutic platform for the ototopical treatment of AOM using an $e x$ vivo chinchilla TM model to study membrane resistivity and small molecule permeation. The liquid state of Poloxamer 407 solutions at room temperature facilitated administration and permitted intimate contact between prepared formulations and the TM. We have demonstrated both sustained ciprofloxacin release out of the gel reservoir in vitro and flux across the TM ex vivo, where incorporation of combination CPEs increases ciprofloxacin flux to therapeutic levels. The gel minimally affected auditory thresholds and induced a minimal inflammatory response in the TM. The ability to prolong antibiotic delivery to the middle ear with a one-time gel administration could potentially improve patient compliance, avoid systemic toxicity and prevent the development of pathogen resistance associated with conventional oral antibiotic therapy.

\section{Acknowledgments}

This work was financially supported by Grant Number R21DC009986 from the National Institute of Deafness and Communicative Disorders (NIDCD).

\section{References}

1. Berman S. Otitis media in children. N Engl J Med. 1995; 332(23):1560-5. [PubMed: 7739711]

2. Freid VM, Makuc DM, Rooks RN. Ambulatory health care visits by children: principal diagnosis and place of visit. Vital Health Stat. 1998; 13(137):1-23.

3. Faden H, Duffy L, Boeve M. Otitis media: back to basics. Pediatr Infect Dis J. 1998; 17(12):110512. [PubMed: 9877357]

4. Bluestone, CD.; Klein, JO. Otitis media in infants and children. BC Decker, Inc; Hamilton: 2007.

5. Pichichero ME. Acute otitis media: part II. Treatment in an era of increasing antibiotic resistance. Am Fam Physician. 2000; 61(8):2410-6. [PubMed: 10794582]

6. Koulich E, Roland PS, Pawlowski KS. Comparison of systemic and otic administration of ofloxacin. Laryngoscope. 2010; 120(10):2083-8. [PubMed: 20830758]

7. Prausnitz MR, Mitragotri S, Langer R. Current status and future potential of transdermal drug delivery. Nat Rev Drug Discov. 2004; 3(2):115-24. [PubMed: 15040576]

8. Prausnitz MR, Langer R. Transdermal drug delivery. Nature Biotechnol. 2008; 26(11):1261-8. [PubMed: 18997767]

9. Walker RB, Smith EW. The role of percutaneous penetration enhancers. Adv Drug Deliv Rev. 1996; 18:295-301.

10. Bauerova K, Matusova D, Kassai Z. Chemical enhancers for transdermal drug transport. Eur J Drug Metab Pharmacokinet. 2001; 26(1-2):85-94. [PubMed: 11554439]

11. Karande P, Jain A, Ergun K, Kispersky V, Mitragotri S. Design principles of chemical penetration enhancers for transdermal drug delivery. Proc Natl Acad Sci U S A. 2005; 102(13):4688-93. [PubMed: 15774584]

12. Bronaugh, RL.; Maibach, HI. Percutaneous absorption : drugs--cosmetics--mechanisms-methodology. 3rd, rev. and expanded. ed.. Marcel Dekker; New York: 1999.

13. Williams, A. Transdermal and topical drug delivery: from theory to clinical practice. Pharmaceutical Press; London: 2003.

14. Guy, RH.; Hadgraft, J. Transdermal drug delivery. Informa HealthCare; 2003. 
15. Kabanov AV, Batrakova EV, Alakhov VY. Pluronic block copolymers as novel polymer therapeutics for drug and gene delivery. J Control Release. 2002; 82(2-3):189-212. [PubMed: 12175737]

16. Moghimi SM, Hunter AC. Poloxamers and poloxamines in nanoparticle engineering and experimental medicine. Trends Biotechnol. 2000; 18(10):412-20. [PubMed: 10998507]

17. Ruel-Gariépy E, Leroux J-C. In situ-forming hydrogels--review of temperature-sensitive systems. Eur J Pharm Biopharm. 2004; 58(2):409-26. [PubMed: 15296964]

18. Wall GM, Stroman DW, Roland PS, Dohar J. Ciprofloxacin 0.3\%/dexamethasone $0.1 \%$ sterile otic suspension for the topical treatment of ear infections. Pediatr Infect Dis J. 2009; 28(2):141. [PubMed: 19116600]

19. Takács-Novák K, Józan M, Hermecz I, Szász G. Lipophilicity of antibacterial fluoroquinolones. Int J Pharm. 1992; 79(1-3):89-96.

20. Schmolka IR. Artificial skin I. Preparation and properties of Pluronic F-127 gels for treatment of burns. J Biomed Mater Res A. 1972; 6(6):571-82.

21. Yun MO, Choi HG, Jung JH, Kim CK. Development of a thermo-reversible insulin liquid suppository with bioavailability enhancement. Int J Pharm. 1999; 189(2):137-45. [PubMed: 10536242]

22. Tang H, Mitragotri S, Blankschtein D, Langer R. Theoretical description of transdermal transport of hydrophilic permeants: application to low-frequency sonophoresis. J Pharm Sci. 2001; 90(5): 545-68. [PubMed: 11288100]

23. Kasting GB, Bowman LA. Electrical analysis of fresh, excised human skin: a comparison with frozen skin. Pharm Res. 1990; 7(11):1141-6. [PubMed: 2293212]

24. Kasting GB, Bowman LA. DC electrical properties of frozen, excised human skin. Pharm Res. 1990; 7(2):134-43. [PubMed: 2308893]

25. El-Kattan AF, Asbill CS, Kim N, Michniak BB. Effect of formulation variables on the percutaneous permeation of ketoprofen from gel formulations. Drug Deliv. 2000; 7(3):147-53. [PubMed: 10989915]

26. Gorukanti SR, Li L, Kim KH. Transdermal delivery of antiparkinsonian agent, benztropine. I. Effect of vehicles on skin permeation. Int J Pharm. 1999; 192(2):159-72. [PubMed: 10567747]

27. Gwak HS, Kim SU, Chun IK. Effect of vehicles and enhancers on the in vitro permeation of melatonin through hairless mouse skin. Arch Pharm Res. 2002; 25(3):392-6. [PubMed: 12135116]

28. Gwak HS, Oh IS, Chun IK. Transdermal delivery of ondansetron hydrochloride: effects of vehicles and penetration enhancers. Drug Dev Ind Pharm. 2004; 30(2):187-94. [PubMed: 15089053]

29. Huang YB, Fang JY, Hung CH, Wu PC, Tsai YH. Cyclic monoterpene extract from cardamom oil as a skin permeation enhancer for indomethacin: in vitro and in vivo studies. Biol Pharm Bull. 1999; 22(6):642-6. [PubMed: 10408241]

30. Kim DD, Chien YW. Transdermal delivery of zalcitabine: in vitro skin permeation study. AIDS. 1995; 9(12):1331-6. [PubMed: 8605052]

31. Mura P, Faucci MT, Bramanti G, Corti P. Evaluation of transcutol as a clonazepam transdermal permeation enhancer from hydrophilic gel formulations. Eur J Pharm Sci. 2000; 9(4):365-72. [PubMed: 10664476]

32. Williams AC, Barry BW. Penetration enhancers. Adv Drug Deliv Rev. 2004; 56(5):603-18. [PubMed: 15019749]

33. Hoare TR, Kohane DS. Hydrogels in drug delivery: progress and challenges. Polymer. 2008; 49(8):1993-2007.

34. Ricci EJ, Lunardi LO, Nanclares DM, Marchetti JM. Sustained release of lidocaine from poloxamer 407 gels. Int J Pharm. 2005; 288(2):235-44. [PubMed: 15620863]

35. Langer R. New methods of drug delivery. Science. 1990; 249(4976):1527. [PubMed: 2218494]

36. Gilbert JC, Hadgraft J, Bye A, Brookes LG. Drug release from Pluronic F-127 gels. Int J Pharm. 1986; 32(2-3):223-8.

37. Hudson SP, Langer R, Fink GR, Kohane DS. Injectable in situ cross-linking hydrogels for local antifungal therapy. Biomaterials. 2010; 31(6):1444-52. [PubMed: 19942285] 
38. Ruel-Gariépy E, Shive M, Bichara A, Berrada M, Le Garrec D, Chenite A, et al. A thermosensitive chitosan-based hydrogel for the local delivery of paclitaxel. Eur J Pharm Biopharm. 2004; 57(1): 53-63. [PubMed: 14729080]

39. Hoffer ME, Balough BJ, Kopke RD, Henderson J, Decicco M, Wester DC, et al. Morphologic changes in the inner ear of chinchilla laniger after middle ear administration of gentamicin in a sustained-release vehicle. Otolaryngol Head Neck Surg. 1999; 120(5):643. [PubMed: 10229587]

40. Sheppard WM, Wanamaker HH, Pack A, Yamamoto S, Slepecky N. Direct round window application of gentamicin with varying delivery vehicles: a comparison of ototoxicity. Otolaryngol Head Neck Surg. 2004; 131(6):890-6. [PubMed: 15577786]

41. Uhde G. The problem of permeability and anesthesia of the tympanic membrane. Arch Otolaryngol Head Neck Surg. 1957; 66(4):391-407.

42. Doyle WJ, Alper CM, Seroky JT, Karnavas WJ. Exchange rates of gases across the tympanic membrane in rhesus monkeys. Acta Otolaryngol. 1998; 118(4):567-73. [PubMed: 9726685]

43. Hoffman RA, Li CL. Tetracaine topical anesthesia for myringotomy. Laryngoscope. 2001; 111(9): 1636-8. [PubMed: 11568619]

44. Karande P, Jain A, Mitragotri S. Insights into synergistic interactions in binary mixtures of chemical permeation enhancers for transdermal drug delivery. J Control Release. 2006; 115(1):8593. [PubMed: 16935378]

45. Karande P, Jain A, Mitragotri S. Relationships between skin's electrical impedance and permeability in the presence of chemical enhancers. J Control Release. 2006; 110(2):307-13. [PubMed: 16313994]

46. Lopez RFV, Seto JE, Blankschtein D, Langer R. Enhancing the transdermal delivery of rigid nanoparticles using the simultaneous application of ultrasound and sodium lauryl sulfate. Biomaterials. 2011; 32(3):933-41. [PubMed: 20971504]

47. Bagul A, Taha R, Metcalfe M, Brook N, Nicholson M. Pre-incision infiltration of local anesthetic reduces postoperative pain with no effects on bruising and wound cosmesis after thyroid surgery. Thyroid. 2005; 15(11):1245-8. [PubMed: 16356087]

48. Tsang M, Guy RH. Effect of aqueous cream BP on human stratum corneum in vivo. Br J Dermatol. 2010; 163(5):954-8. [PubMed: 20649794]

49. Karande P, Mitragotri S. Enhancement of transdermal drug delivery via synergistic action of chemicals. Biochim Biophys Acta. 2009; 1788(11):2362-73. [PubMed: 19733150]

50. Karande P, Arora A, Pham TK, Stevens D, Wojicki A, Mitragotri S. Transcutaneous immunization using common chemicals. J Control Release. 2009; 138(2):134-40. [PubMed: 19426770]

51. Chen-Chow PC, Frank SG. In vitro release of lidocaine from Pluronic F-127 gels. Int J Pharm. 1981; 8(2):89-99.

52. Veyries ML, Couarraze G, Geiger S, Agnely F, Massias L, Kunzli B, et al. Controlled release of vancomycin from poloxamer 407 gels. Int J Pharm. 1999; 192(2):183-93. [PubMed: 10567749]

53. Yong CS, Choi JS, Quan QZ, Rhee JD, Kim CK, Lim SJ, et al. Effect of sodium chloride on the gelation temperature, gel strength and bioadhesive force of poloxamer gels containing diclofenac sodium. Int J Pharm. 2001; 226(1-2):195-205. [PubMed: 11532582]

54. Juhasz J, Lenaerts V, Raymond P, Ong H. Diffusion of rat atrial natriuretic factor in thermoreversible poloxamer gels. Biomaterials. 1989; 10(4):265-8. [PubMed: 2525935]

55. Liu T, Chu B. Formation of homogeneous gel-like phases by mixed triblock copolymer micelles in aqueous solution: FCC to BCC phase transition. J Appl Cryst. 2000; 33:727-30.

56. Vadnere M, Amidon G, Lindenbaum S, Haslam JL. Thermodynamic studies on the gel-sol transition of some pluronic polyols. Int J Pharm. 1984; 22(2-3):207-18.

57. Gilbert JC, Richardson JL, Davies MC, Palin KJ, Hadgraft J. The effect of solutes and polymers on the gelation properties of Pluronic F-127 solutions for controlled drug delivery. J Control Release. 1987; 5(2):113-8.

58. Ikiz AÖ, Serbetçioglu B, Güneri EA, Sütay S, Ceryan K. Investigation of topical ciprofloxacin ototoxicity in guinea pigs. Acta Otolaryngol. 1998; 118(6):808-12. [PubMed: 9870624]

59. Brownlee RE, Hulka GF, Prazma J, Pillsbury HC III. Ciprofloxacin: use as a topical otic preparation. Arch Otolaryngol Head Neck Surg. 1992; 118(4):392. [PubMed: 1554468] 
60. Daniel HJ 3rd, Fulghum RS, Brinn JE, Barrett KA. Comparative anatomy of eustachian tube and middle ear cavity in animal models for otitis media. Ann Otol Rhinol Laryngol. 1982; 91(1 Pt 1): 82-9. [PubMed: 7073182]

61. Vrettakos PA, Dear SP, Saunders JC. Middle ear structure in the chinchilla: a quantitative study. Am J Otolaryngol. 1988; 9(2):58-67. [PubMed: 3400821] 
A
B

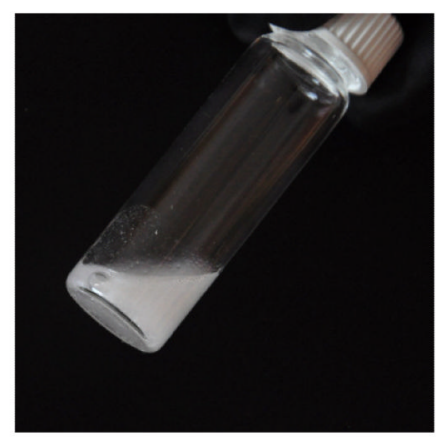

$37^{\circ} \mathrm{C}$

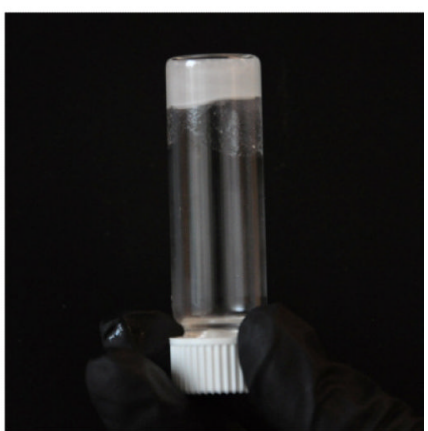

Figure 1.

(A) Schematic representation of the in-situ forming antibiotic-loaded P407 gel at the tympanic membrane (TM). Antibiotic diffuses out of the gel, across the TM, and into the infected middle ear cavity. (B) Liquid-to-gel transition of 18\% P407-3CPE formulation at $37^{\circ} \mathrm{C}$. 


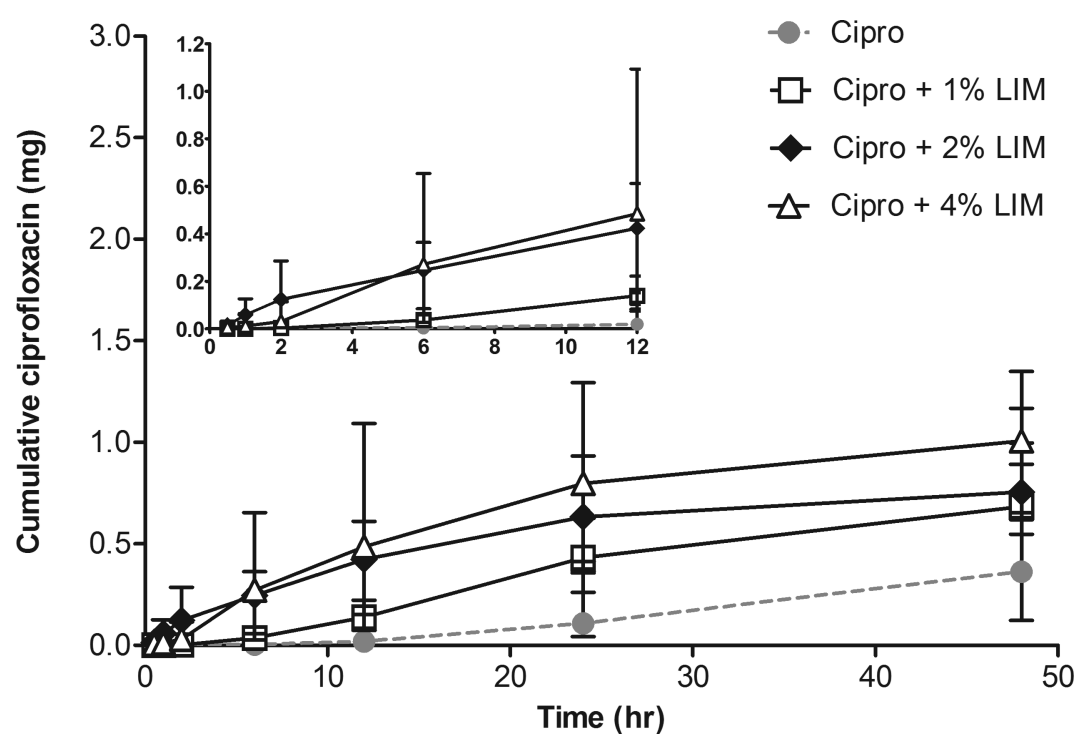

Figure 2.

Effect of concentration of a single CPE, limonene (LIM), on ciprofloxacin permeation across the chinchilla TM. Inset: Ciprofloxacin permeation in the first 12 hours. $2 \mathrm{mg}$ of ciprofloxacin were originally loaded into each hydrogel. Values are medians \pm interquartile ranges $(n=4)$. 


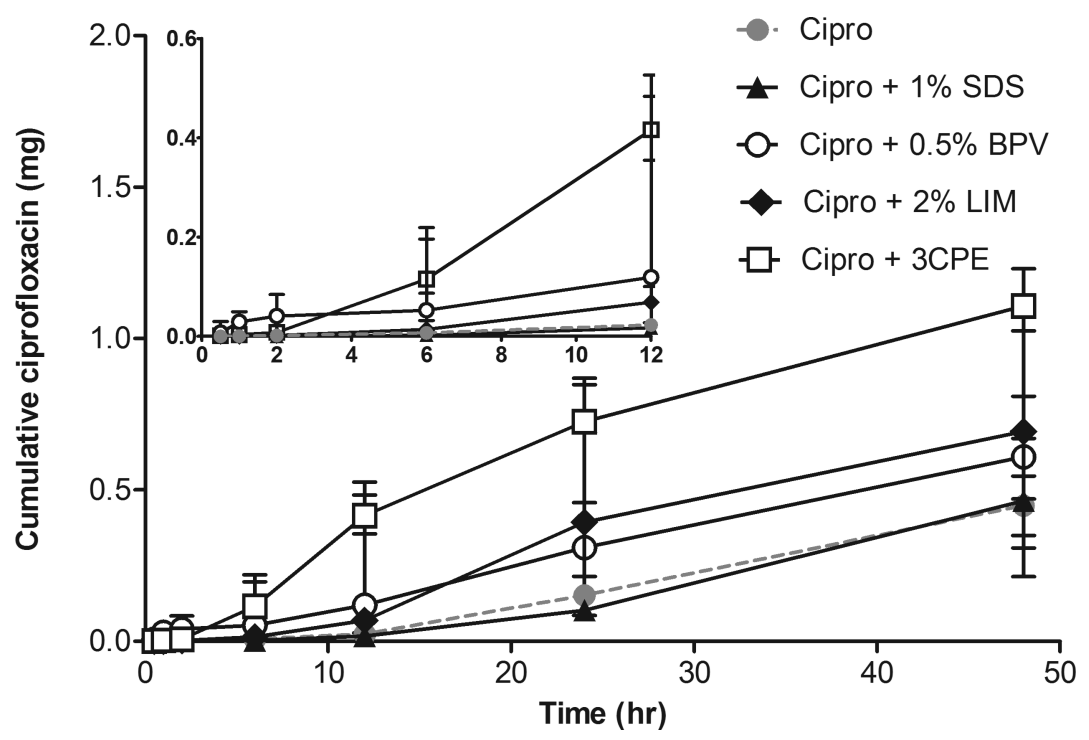

Figure 3.

Cumulative ciprofloxacin permeation across chinchilla TMs from solutions with single and combination CPEs. SDS = sodium dodecyl sulfate, BPV = bupivacaine, $\mathrm{LIM}=$ limonene. 2 $\mathrm{mg}$ of ciprofloxacin were loaded into each sample. Inset: Ciprofloxacin permeation profiles in the first 12 hours. Values are medians \pm interquartile ranges $(n=4)$. 


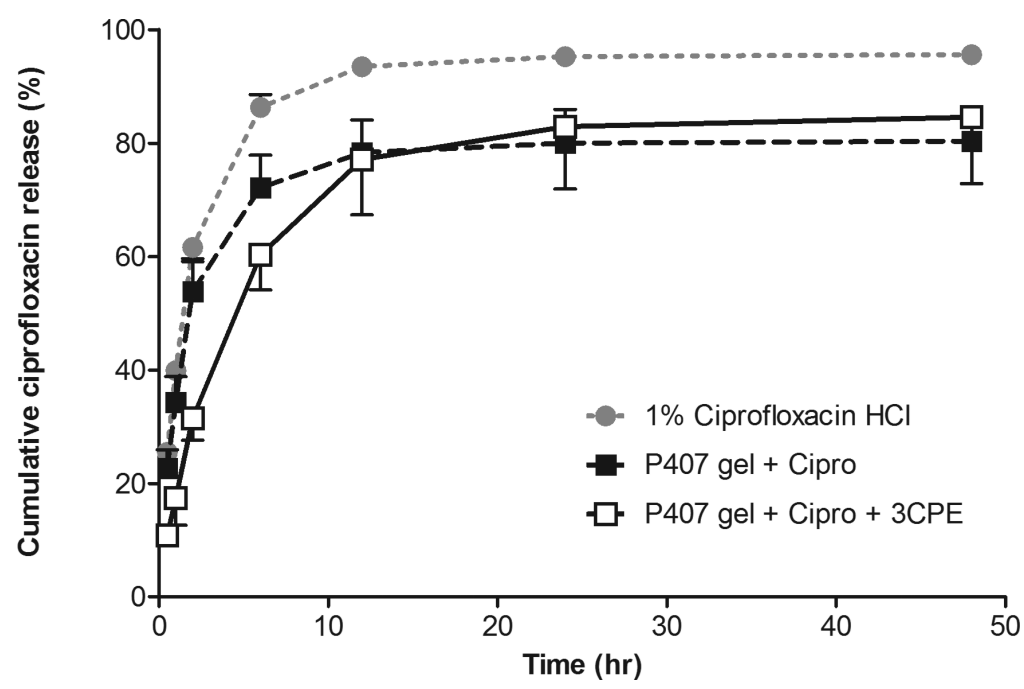

Figure 4.

Cumulative ciprofloxacin release from $18 \%$ P407 gels at $37{ }^{\circ} \mathrm{C}$ with and without $3 \mathrm{CPE} .2$ $\mathrm{mg}$ of ciprofloxacin were originally loaded into each hydrogel. Values are medians \pm interquartile ranges $(n=4)$. 


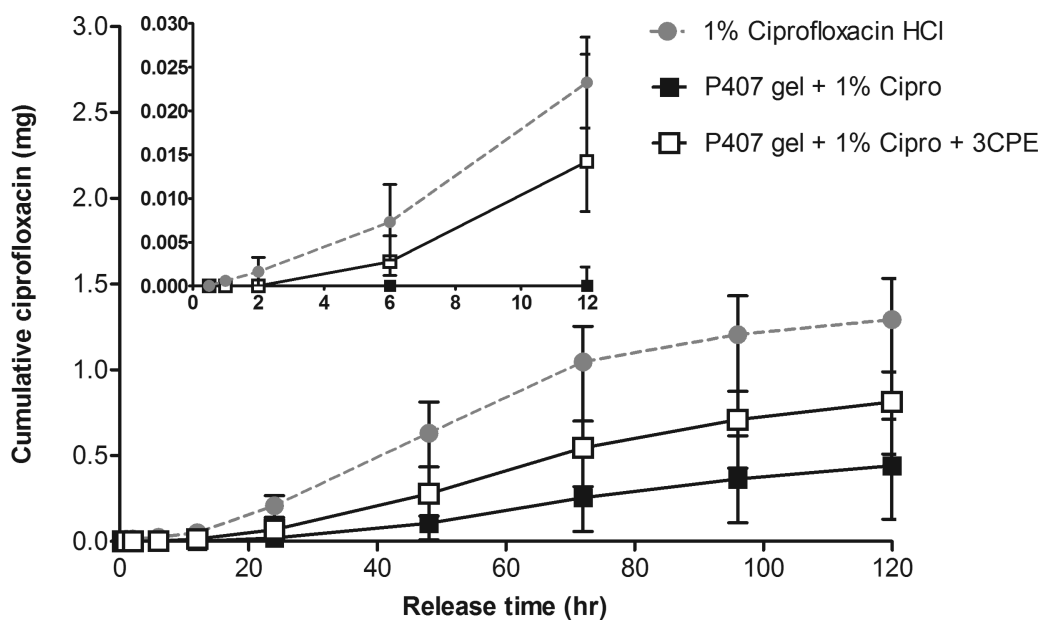

Figure 5.

Cumulative ciprofloxacin permeation across the chinchilla TM from gels containing $18 \%$ P407 and 3CPE at $37^{\circ} \mathrm{C}$. Inset: Ciprofloxacin permeation profiles in the first 12 hours. $2 \mathrm{mg}$ of ciprofloxacin was originally loaded into each hydrogel. Values are medians \pm interquartile range. $(n=6)$. 


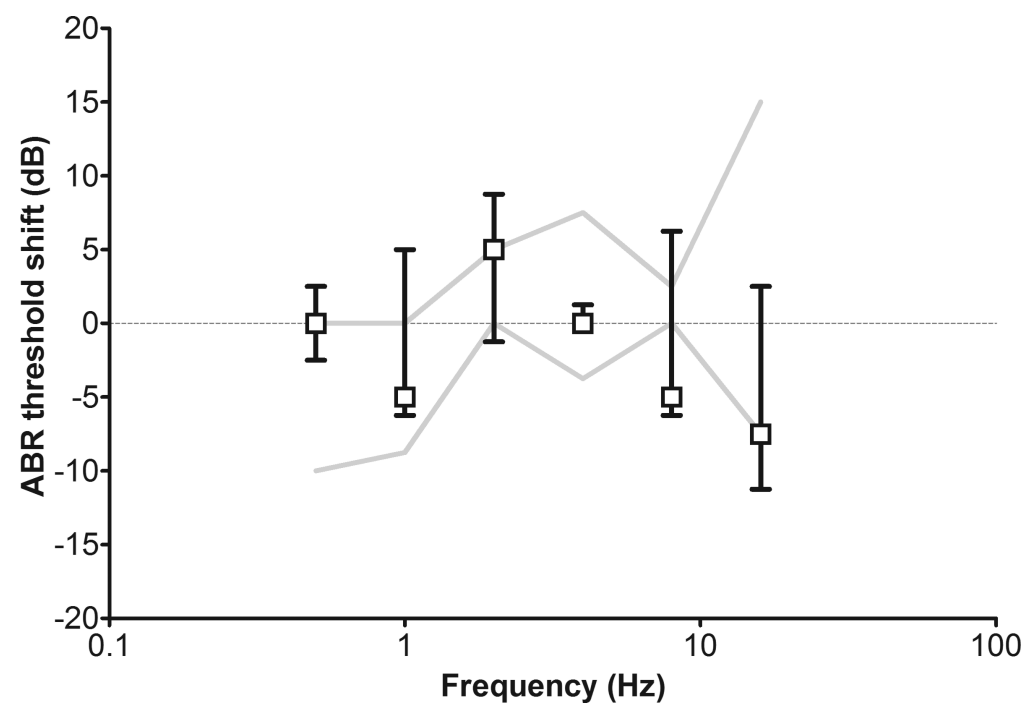

Figure 6.

ABR threshold shifts at a range of frequencies immediately following application of $18 \%$ P407 hydrogels containing CPEs. The dotted line indicates where data would lie if there was no change in ABR thresholds compared to pre-application measurements. The grey lines indicate the observed interquartile spread of pre-application control measurements $(n=10)$. Experimental values are medians \pm interquartile range $(n=4)$. 


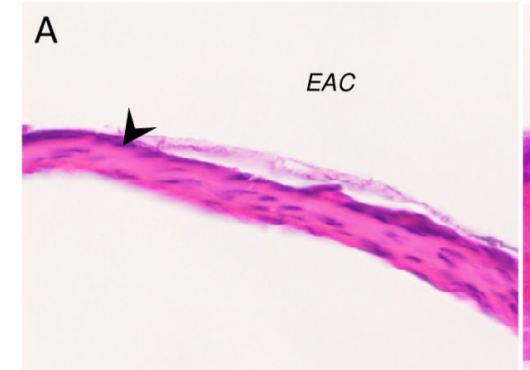

B
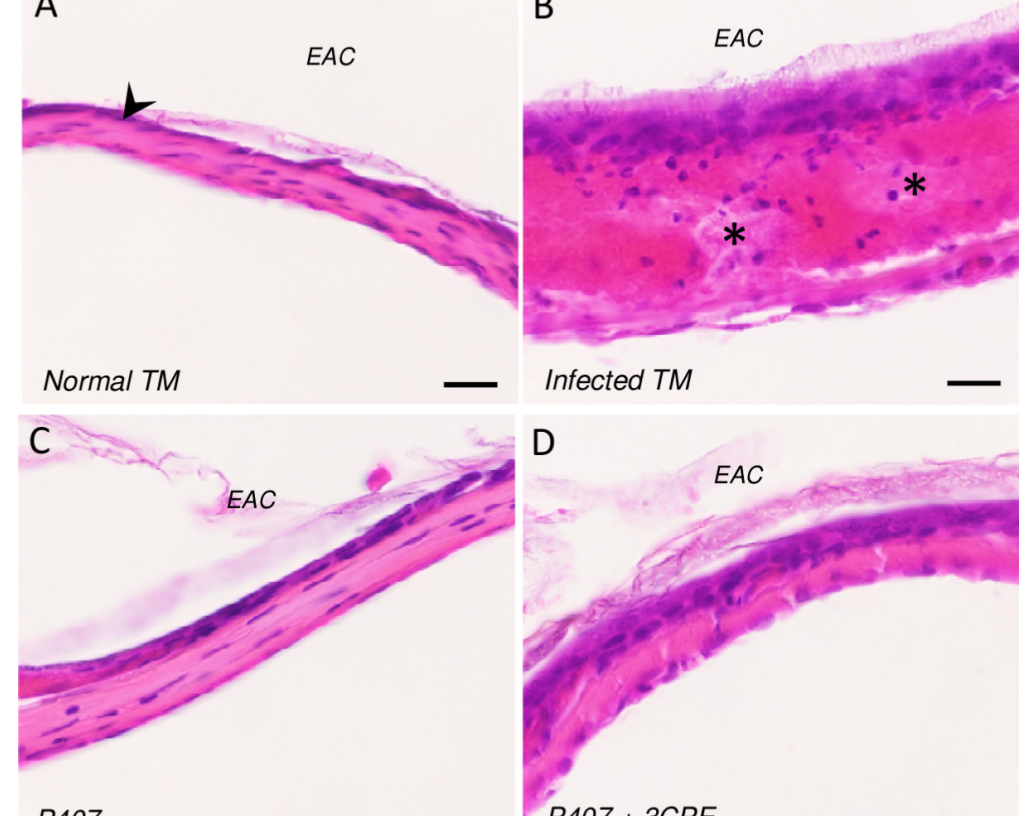

Infected TM

D

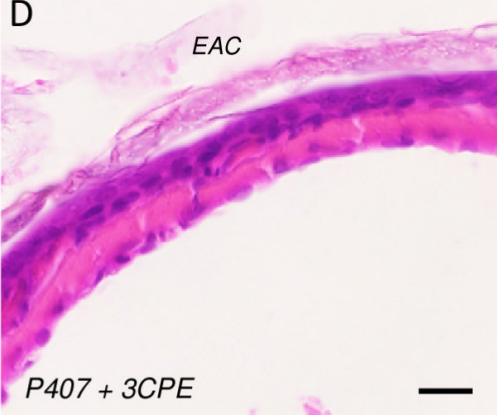

Figure 7.

Photomicrographs of hematoxylin and eosin-stained chinchilla tympanic membrane (TM) sections $(n=2)$. (A) Normal, untreated TM with distinct stratum corneum (SC) layer (arrow). (B) Infected TM is markedly thicker and exhibits acute inflammation with interstitial edema and cellular infiltration (asterisk). (C, D) TMs exposed to gels containing (C) ciprofloxacin or (D) ciprofloxacin and 3CPE for 7 days showed slight thickening with no or minimal inflammation. EAC $=$ external auditory canal. Magnification, 400X. Scale bar $=20 \mu \mathrm{m}$. 
Table 1

Gelation properties of various $\mathrm{P} 407$ hydrogel formulations ${ }^{a}$.

\begin{tabular}{|c|c|c|c|}
\hline & Poloxamer 407 concentration (\%) & Gelation temperature $\left({ }^{\circ} \mathbf{C}\right)$ & Gelation time $^{b}(\mathrm{~s})$ \\
\hline \multirow[t]{3}{*}{ No CPE } & 16 & 41.0 & $>90$ \\
\hline & 18 & 38.5 & 42.0 \\
\hline & 20 & 29.5 & 19.5 \\
\hline \multirow[t]{3}{*}{$3 C P E^{c}$} & 16 & 27.0 & 90.0 \\
\hline & 18 & 24.5 & 15.0 \\
\hline & 20 & 23.0 & 9.0 \\
\hline $\begin{array}{l}\text { All formu } \\
\text { At } 37^{\circ} \mathrm{C} \text {. }\end{array}$ & tions contain $1 \%$ ciprofloxacin $\mathrm{HCl}$ & $=4)$. & \\
\hline
\end{tabular}

